\title{
Enhanced Euclid Algorithm for Modular Multiplicative Inverse and Its Application in Cryptographic Protocols
}

\author{
Boris S. Verkhovsky \\ Computer Science Department, New Jersey Institute of Technology, \\ University Heights, Newark, USA \\ E-mail: verb@njit.edu \\ Received August 21, 2010; revised October 4, 2010; accepted November 16, 2010
}

\begin{abstract}
Numerous cryptographic algorithms (ElGamal, Rabin, RSA, NTRU etc) require multiple computations of modulo multiplicative inverses. This paper describes and validates a new algorithm, called the Enhanced Euclid Algorithm, for modular multiplicative inverse (MMI). Analysis of the proposed algorithm shows that it is more efficient than the Extended Euclid algorithm (XEA). In addition, if a MMI does not exist, then it is not necessary to use the Backtracking procedure in the proposed algorithm; this case requires fewer operations on every step (divisions, multiplications, additions, assignments and push operations on stack), than the XEA. Overall, XEA uses more multiplications, additions, assignments and twice as many variables than the proposed algorithm.
\end{abstract}

Keywords: Extended-Euclid Algorithm, Modular Multiplicative Inverse, Public-Key Cryptography, RSA Cryptocol, Rabin Information Hiding Algorithm, ElGamal Encryption/Decryption, NTRU Cryptosystem, Computer Simulation, Low Memory Devices

\section{Introduction}

Elements of modular arithmetic are essential for public-key encryption algorithms such as the RSA cryptographic algorithm [1-3], and RSA with digital signature [4]; ElGamal cryptocol [5]; Rabin encryption/decryption scheme based on extraction of square roots [6]; NTRU cryptosystem [7]; and extensions of some of these algorithms in Gaussian arithmetic require computation of a modular multiplicative inverse (MMI) [8-10]. The MMI is also computed for cryptanalysis of public-key cryptographic protocols [11-13].

A new algorithm, called the Enhanced-Euclid Algorithm (NEA), for modular multiplicative inverse (MMI) is described and validated in this paper.

Definition 1: Given relatively prime integers $p_{0}$ and $p_{1}$, there exists an unique integer $x$ such that

$$
p_{1} x=1\left(\bmod p_{0}\right) .
$$

Then $x$ is defined as the modular multiplicative inverse of $p_{1}$ modulo $p_{0}$ or, for short, MMI.

The NEA finds for two relatively prime integers $p_{0}$ and $p_{1}$ an integer number $x$ that satisfies the Equation (1). And if $p_{0}$ and $p_{1}$ are not relatively prime, then
NEA finds a $\operatorname{gcd}\left(p_{0}, p_{1}\right)$. The Extended-Euclid algorithm (XEA) also finds a MMI of $p_{1}$ modulo $p_{0}$ if and only if $\operatorname{gcd}\left(p_{0}, p_{1}\right)=1[1,2,7]$.

This paper proves the validity of NEA and provides its analysis. The analysis demonstrates that NEA is faster than the Extended Euclid algorithm. Preliminary results of this paper are published in [8].

\section{Basic Arrays and Their Properties}

Let's consider five finite integer arrays:

$$
\left\{p_{i}\right\} ;\left\{c_{i}\right\} ;\left\{t_{k}\right\} ;\left\{w_{k}\right\} ;\left\{z_{k}\right\}
$$

Definition 2: Let $\left\{p_{i}\right\}$ and $\left\{c_{i}\right\}$ be integer arrays defined according to the following generating rules:

Given two relatively prime integers $p_{0}$ and $p_{1}$ such that $p_{0}>p_{1}$, for $i \geq 1$ do while $p_{i} \geq 2$,

$$
p_{i+1}:=p_{i-1} \bmod p_{i} ; c_{i}:=\left\lfloor p_{i-1} / p_{i}\right\rfloor .
$$

Definition 3: Let $\left\{t_{k}\right\}$ be an arbitrary integer array for all $k \geq 1$; let for initially specified $w_{0}, w_{1}, z_{0}$ and $z_{1}$ the following generating rules be defined for all $k \geq 2$ : 


$$
w_{k}:=w_{k-1} t_{k-1}+w_{k-2}
$$

and

$$
z_{k}:=z_{k-1} t_{k-1}+z_{k-2} \text {. }
$$

Proposition 1: Let's define for $k \geq 1$

$$
D_{k}:=\left|\begin{array}{ll}
w_{k} & w_{k-1} \\
z_{k} & z_{k-1}
\end{array}\right| .
$$

Then

$$
D_{k}=(-1)^{k-1} D_{1} \text {. }
$$

Proof: Consider $D_{k}$ and substitute in the left column the values of $w_{k}$ and $z_{k}$ defined in (4). After simplifications we derive that $D_{k}=-D_{k-1}$, which recursively implies (6).

Proposition 2: Consider the integer arrays $\left\{t_{k}\right\}$, $\left\{w_{k}\right\}$, and $\left\{z_{k}\right\}$ (4) where $w_{0}:=1 ; z_{0}:=0 ;\left|z_{1}\right|:=1$.

Then $(-1)^{k-1} z_{1} z_{k}$ is a multiplicative inverse of $w_{k-1}$ modulo $w_{k}$ for every $w_{1}$.

Proof: Indeed, since $D_{1}=-z_{1}$, then (5) implies that

$$
w_{k} z_{k-1}-z_{k} w_{k-1}=(-1)^{k} z_{1} \text {, }
$$

or that

$$
w_{k-1}\left[(-1)^{k-1} z_{1} z_{k}\right]-z_{1}^{2}=w_{k}\left[(-1)^{k-1} z_{1} z_{k-1}\right]
$$

Then from (7) it follows that

$$
\begin{aligned}
& {\left[w_{k-1}(-1)^{k-1} z_{1} z_{k}-1\right] / w_{k}=(-1)^{k-1} z_{1} z_{k-1} \text {, } } \\
& \text { i.e., } x=(-1)^{k-1} z_{1} z_{k} .
\end{aligned}
$$

Proposition 3: If for all $0 \leq k \leq r$

$$
t_{k}:=c_{r-k} \text {, and } w_{k}:=p_{r-k},
$$

then $p_{0}$ and $p_{1}$ are the initial values that generate the arrays $\left\{p_{i}\right\},\left\{c_{i}\right\}$,

$$
\left\{t_{k}\right\}:=\left\{c_{r-k}\right\} \text { and }\left\{w_{k}\right\}:=\left\{p_{r-k}\right\} \text {. }
$$

Remark 1: Notice that $\left\{t_{k}\right\}=\left\{c_{i}\right\}^{R}$ and $\left\{w_{k}\right\}=\left\{p_{i}\right\}^{R}$, where the superscript $R$ means that the arrays $\left\{c_{i}\right\}$ and $\left\{p_{i}\right\}$ are written in a reverse order.

Theorem 1: For all $k=1, \cdots, r,(-1)^{k-1} z_{1} z_{k}$ is the inverse of $p_{r-k+1}$ modulo $p$.

Proof follows from Propositions 1-3.

Theorem 1 implies the following assertions:

1) if $k$ is odd and $z_{1}=1$,

then $x:=z_{k}>0$;

2) if $k$ is even and $z_{1}=-1$,

then $x:=z_{k}<0$.

In the latter case select

$$
x:=p_{0}+z_{k} .
$$

\section{Enhanced Euclid Algorithm for MMI}

The proposed algorithm uses stack as a data structure, [2].

vars: $r ; L ; M ; S$; $t$ : all integer numbers; $b$ : Boolean; procedure Forward:

$$
\begin{gathered}
L:=p_{0} ; M:=p_{1} ; b:=0 ;\{r:=0\} ; \\
\text { repeat } t:=\lfloor L / M\rfloor ; S:=L-M t ; \\
b:=1-b ;\{r:=r+1\} ;
\end{gathered}
$$

push $t$ \{onto the top of the stack\};

$$
L:=M ; M:=S \text {; }
$$

until $S=1$;

Remark 2: if $S=0$, then $\operatorname{gcd}\left(p_{0}, p_{1}\right)=t$; therefore the $M M I$ does not exist;

procedure Backtracking:

$S:=0 ; \quad M:=(-1)^{b} ;\{$ by (9) in the Theorem 1$\}$;

repeat pop $t$ from the top of the stack\};

$$
L:=M t+S ; S:=M ; M:=L ;
$$

until the stack is empty;

output $x$ : $=L$; $\left\{\right.$ if $x<0$, then $\left.x:=x+p_{0}\right\}$.

Remark 3: $r$ is the height of the stack and is used below for analysis of the proposed algorithm.

\section{Two Illustrative Examples}

Let's demonstrate how NEA finds a multiplicative inverse $x$ of 27,182,845 modulo 31,415,926. Table 1 below shows the computation of remainders in the upper row and stores the quotients in the middle row (the stack). Then the Backtracking procedure is used to compute from right to left until the stack is empty. The inputs and MMI are shown in bold, and the stack values are in italics. Since the total number of steps (the height of the stack) is equal to fourteen (i.e., even), then $x=13,939,773$.

Direct verification confirms that indeed

$$
27182845 * 13939773 \bmod 31415926=1 .
$$

In the second numerical example we need to find the MMI $z$ of 27,319,913 modulo 177,276,627 see Table 2 \}. Since the height of the stack is odd, then

$$
z=177276627-34480855=\mathbf{1 4 2 7 9 5 7 7 2} .
$$

Direct computation verifies that $z$ is indeed the MMI of 27319913, since

$$
27319913 * 142795772 \bmod 177276627=1 .
$$

\section{Complexity Analysis of MMI Algorithm}

Consider four non-negative integer arrays $\left\{q_{k}\right\},\left\{d_{k}\right\}$, 
Table 1. Modular multiplicative inverse algorithm in progress.

\begin{tabular}{|c|c|c|c|c|c|c|c|c|c|c|}
\hline 31415926 & 27182845 & & 4233081 & \multicolumn{2}{|c|}{1784359} & 664363 & \multicolumn{2}{|r|}{455633} & \multicolumn{2}{|c|}{208730} \\
\hline Stack & 1 & & 6 & \multicolumn{2}{|c|}{2} & 2 & \multicolumn{2}{|r|}{1} & \multicolumn{2}{|c|}{2} \\
\hline 13939773 & 12061484 & & 1878289 & \multicolumn{2}{|c|}{791750} & 294789 & \multicolumn{2}{|r|}{202172} & \multicolumn{2}{|c|}{92617} \\
\hline \multicolumn{11}{|c|}{ (continuation) } \\
\hline 208730 & 38173 & 17865 & & 2443 & 764 & 151 & 9 & 7 & 2 & 1 \\
\hline 2 & 5 & 2 & & 7 & 3 & 5 & 16 & 1 & 3 & $* * *$ \\
\hline 92617 & 16938 & 7927 & & 1084 & 339 & 67 & 4 & 3 & 1 & 0 \\
\hline
\end{tabular}

Table 2. Second numerical illustration.

\begin{tabular}{ccccccccc}
\hline $\mathbf{1 7 7 2 7 6 6 2 7}$ & $\mathbf{2 7 3 1 9 9 1 3}$ & 13357149 & 605615 & 33619 & 473 & 36 & 5 & 1 \\
Stack & 6 & 2 & 22 & 18 & 71 & 13 & 7 & $* * *$ \\
$\mathbf{3 4 4 8 0 8 5 5}$ & 5313808 & 2598007 & 117794 & 6539 & 92 & 7 & $\mathbf{1}$ & $\mathbf{0}$ \\
\hline
\end{tabular}

$\left\{p_{i}\right\}$ and $\left\{c_{i}\right\}$ defined as follows:

$$
d_{k}:=\left\lfloor q_{k-1} / q_{k}\right\rfloor ;
$$

$\left\{p_{i}\right\}$ and $\left\{c_{i}\right\}$ satisfy (3) and are defined as

$$
p_{i+1}:=p_{i-1}-p_{i} c_{i} ; q_{k+1}:=q_{k-1}-q_{k} d_{k}
$$

Then (3) and (14) imply that

$$
p_{i+1}:=p_{i-1}-p_{i} c_{i}=p_{i-1} \bmod p_{i}
$$

Hence both arrays $\left\{p_{i}\right\}$ and $\left\{q_{k}\right\}$ are strictly decreasing, and all terms of the corresponding arrays $\left\{c_{i}\right\}$ and $\left\{d_{k}\right\}$ are positive integer numbers.

Definition 4: $\left\{x_{j}\right\}_{s}$ is a $(s+1)$-dimensional vector, consisting of the first $s+1$ terms of an array $x_{0}, x_{1}, \ldots, x_{j-1}, x_{j}, \ldots$, i.e.,

$$
\left\{x_{j}\right\}_{s}:=\left(x_{0}, x_{1}, \ldots, x_{s-1}, x_{s}\right) .
$$

Theorem 2: Consider

$$
\left\{c_{i}\right\}_{r} \geq 1 ;\left\{p_{i}\right\}_{s} ;\left\{d_{k}\right\}_{s} ;\left\{q_{k}\right\}_{s} \geq 1
$$

and $\left\{p_{i}\right\}_{r} \geq 1$.

Let $p_{0}=q_{0} ;\left\{c_{i}\right\}_{s} \leq\left\{d_{k}\right\}_{s}$, i.e., for all $j=1, \cdots, s$ there exists at least one $j=l$ such that $c_{l}<d_{l}$. Then for all $1 \leq j \leq s$ the following inequalities hold:

$$
\text { if } 1 \leq j \leq l-1 \text {, then } p_{j} \geq q_{j}
$$

otherwise

$$
p_{j}>q_{j} .
$$

Proof: Assuming that the statement (15) holds for all $i \leq j-1$, it can also be demonstrated by induction that (15) also holds for $i=j$.

Consider

$$
\begin{aligned}
& t_{j}=d_{j}-c_{j}=\left\lfloor q_{j-1} / q_{j}\right\rfloor-\left\lfloor p_{j-1} / p_{j}\right\rfloor \\
& \leq\left\lfloor p_{j-1} / q_{j}\right\rfloor-\left\lfloor p_{j-1} / p_{j}\right\rfloor .
\end{aligned}
$$

If $j \leq l-1$, then $t_{j} \geq 0$ else $t_{j}>0$. Hence from (16) it follows: if $j \leq l-1$, then $p_{j} \geq q_{j}$ otherwise $p_{j}>q_{j}$. Since $p_{0}=q_{0}$, then (14) holds for all $j \leq s$.

Q.E.D.

Consider a pair of relatively prime seeds $p_{0}$ and $p_{1}$ that generates an array $\left\{c_{i}\right\}_{r}=1$. Consider also another pair of relatively prime seeds $p_{0}$ and $q_{1}$ that generates an array $\left\{d_{k}\right\}_{s} \geq 1$, i.e. such that not all its terms are equal to one. Let $r$ and $s$ be the number of steps required respectively to find MMIs for the first and the second pair using either XEA or NEA. This assumption implies that $q_{s}=1$. Then by Theorem $2\left\{p_{i}\right\}_{s} \geq\left\{q_{k}\right\}_{s}$ and $p_{s}>q_{s}=1$. Hence $r>s$.

Corollary: A pair of seeds, that for a given $p_{0}$ requires the maximal number of steps for computation of a MMI, generates an unary array of quotients, i.e., all components in $\left\{c_{i}\right\}_{r}=1$. Thus, as it follows from (3) and (14), this pair of seeds must generate the following array of integer numbers: $p_{2}:=p_{0}-p_{1} ; p_{3}:=p_{1}-p_{2} ; \cdots$; $p_{r}:=p_{r-2}-p_{r-1}=1$. It is easy to verify that this array is equivalent to the sequence of Fibonacci numbers

$$
\left\{F_{r+2}, F_{r+1}, \ldots, F_{4}, F_{3}, F_{2}\right\} \text {. }
$$

In other words, for every $i=0, \ldots, r \quad p_{i}:=F_{r+2-i}$, [14].

Remark 4: The pair $p_{0}=F_{r+2} ; \quad p_{1}=F_{r+1}$ is not the only one that generates a) a unary array of quotients and b) a decreasing integer array where the $r^{\text {th }}$ remainder equals one.

Indeed, the following pairs of seeds have the same 
property for all non-negative integer numbers $t$ and $u$ :

1) $p_{0}=F_{r+2}+t F_{r} ; \quad p_{1}=F_{r+1}+t F_{r-1} ;$ for $t=1$ $\left\{p_{i}\right\}^{R}=\left\{L_{1} ; L_{2} ; \cdots ; L_{r+1}\right\}$ is a sequence of the Lucas numbers $1,3,4,7,11,18, \cdots[15]$;

2) $p_{0}=t F_{r+2}+(1-t) F_{r-1} ; t \geq 1$;

$p_{1}=t F_{r+1}+(1-t) F_{r-2}$

3) $p_{0}=F_{r+1}+t F_{r} ; \quad t \geq 1 ; \quad p_{1}=F_{r}+t F_{r-1}$;

4) $p_{0}=(1+t) F_{r+2}+t F_{r-2}+u F_{r}$;

$p_{1}=(1+t) F_{r+1}+t F_{r-3}+u F_{r-1}$.

Here the Fibonacci numbers with zero and negative indices are computed in accordance with the formula:

$$
F_{-m}=(-1)^{m-1} F_{m} .
$$

For all pairs, listed above, exactly $r$ steps are required to find the MMI. However, all these pairs are special cases of a pair of seeds where

$$
p_{0}=b F_{r}+F_{r-1} \text { and } p_{1}=b F_{r-1}+F_{r-2} \text {. }
$$

Therefore for all $0 \leq i \leq r$

$$
p_{i}=b F_{r-i}+F_{r-i-1}, \quad p_{r-1}=b \text {; and } p_{r}=1 .
$$

Consider

$$
v=(1-\sqrt{5}) / 2 \text { and } w=(1+\sqrt{5}) / 2 .
$$

Using a $z$-transform approach, we deduce that for all $0 \leq k \leq r$

$$
p_{r-k}=\left[(b-v) w^{k}+(w-b) v^{k}\right] / \sqrt{5} .
$$

Then for a large $r$

$$
p_{r-k} \sqrt{5}-(b-v) w^{k}=(w-b)(-1)^{k}|v|^{k} \rightarrow 0
$$

since

$$
|v|<1
$$

The relation (18) implies that for a large $r$

$$
p_{0}=\left[w^{r}(b-v) / \sqrt{5}\right][1+o(w)] .
$$

Let $z:=\max r\left(p_{0}, b\right)=r\left(p_{0}, 2\right)$.

Therefore $^{b \geq 2}$

$$
z=\log _{w}\left[\sqrt{5} p_{0} /(2-v)\right]=\left\lfloor\log _{w} p_{0}\right\rfloor\left[1+o\left(p_{0}\right)\right]
$$

Thus (20) implies that the height of a stack satisfies the following inequality:

$$
r \leq\left\lfloor\log _{w} p_{0}\right\rfloor \times\left[1+o\left(p_{0}\right)\right],[8] .
$$

Example 3: In this example (see the table below) $1919^{-1} \bmod 3105=1364$.

Indeed, $1919 * 1364 \bmod 3105=1$.

Table 3 illustrates the case where the height of the stack is approaching the upper bound in the Inequality (21).

Remark 5: Although this upper bound is achievable if $p_{0}=b F_{r}+F_{r-1}$ and $p_{1}=b F_{r-1}+F_{r-2}$, for this pair of inputs the $M M I$ can be computed explicitly: indeed, it equals $(-1)^{r-1} F_{r}$.

Remark 6: If in RSA public-key encryption [3], $p_{0}=c \times 10^{100}$, then $r \leq 100 / \log _{10} w+\log _{10} c$; therefore $r \leq 479+\log c$.

Over one thousand computer simulations demonstrate that the average height of the stack is actually almost $40 \%$ smaller than the upper bound in (21).

\section{Extended-Euclid Algorithm (XEA)}

XEA also finds a multiplicative inverse of $p_{1}$ modulo $p_{0}$ provided that $\operatorname{gcd}\left(p_{0}, p_{1}\right)=1$, [2,7].

1) Assign $(X 1, X 2, X 3):=\left(1,0, p_{0}\right) ;(Y 1, Y 2, Y 3):=\left(0,1, p_{1}\right)$;

2) if $Y 3=0$ return $X 3=\operatorname{gcd}\left(p_{0}, p_{1}\right)$; $\{$ there is no inverse $\}$;

3) if $Y 3=1$ return $Y 3=\operatorname{gcd}\left(p_{0}, p_{1}\right)$;

else $Y 2$ is the multiplicative inverse;

4)

$$
Q:=\lfloor X 3 / Y 3\rfloor
$$

5) $(T 1, T 2, T 3):=(X 1-Q Y 1, X 2-Q Y 2, X 3-Q Y 3)$;

6)

$$
(X 1, X 2, X 3):=(Y 1, Y 2, Y 3) \text {; }
$$

7)

$$
(Y 1, Y 2, Y 3):=(T 1, T 2, T 3) \text {; }
$$

8) goto 2 .

\section{Comparative analysis of NEA vs. XEA}

Both algorithms require equal number of steps $r$ for computation of all quotients: values of $t$ on the Forward procedure in (10), and $Q$ in (22), respectively.

In addition, NEA requires $r$ more steps on the Backtracking procedure to compute the values of $L$ in (12).

Therefore each step of XEA requires one division, three multiplications, three long algebraic additions and ten assignments, see (22)-(25).

Table 3. $\{$ Worst-case space complexity\}: Size of stack.

\begin{tabular}{cccccccccccccccccc}
\hline $\mathbf{3 1 0 5}$ & $\mathbf{1 9 1 9}$ & 1186 & 733 & 453 & 280 & 173 & 107 & 66 & 41 & 25 & 16 & 9 & 7 & 2 & 1 \\
Stack & 1 & 1 & 1 & 1 & 1 & 1 & 1 & 1 & 1 & 1 & 1 & 1 & 1 & 3 & $* * *$ \\
$\mathbf{1 3 6 4}$ & 843 & 521 & 322 & 199 & 123 & 76 & 47 & 29 & 18 & 11 & 7 & 4 & 3 & $\mathbf{1}$ & $\mathbf{0}$ \\
\hline
\end{tabular}


XEA uses ten variables. Yet in both procedures NEA uses one division, two multiplications, two long additions, two stack operations, (push and pop), and eight assignments, see (10)-(12). NEA uses four integer variables, one binary variable and, in addition, $\Theta\left(\log _{w} p_{0}\right)$ of memory to store the stack.

Notice that if a MMI does not exist, then there is no need to use the Backtracking procedure in NEA. In this case NEA requires even fewer operations than XEA: one division, one multiplication, one addition, one push operation and five assignments per every step. Yet XEA still requires the same number of operations per step as in the case if a MMI does exist. Hence, overall XEA uses more multiplications, more additions, more assignments and twice as many variables than the proposed algorithm.

\section{Average Complexity of XEA and NEA}

If both inputs $p_{0}$ and $p_{1}$ are chosen randomly, then the probability that $\operatorname{gcd}\left(p_{0}, p_{1}\right)=1$ equals $6 / \pi^{2}$ [2].

Let us consider the following notations:

$w_{\text {xea }}$-worst-case specific complexity (per step) of XEA;

$w_{\text {nea }}$-worst-case specific complexity of NEA;

$a_{\text {xea }}$-average-case specific complexity of XEA;

$a_{\text {nea }}$-average-case specific complexity of NEA;

let $t_{d} ; t_{m} ; t_{a} ; t_{s} ; t_{s t}$ be time complexities of division, multiplication, addition, assignment and stack operations push and pop respectively.

Then

$$
\begin{gathered}
w_{\text {xеa }}=t_{d}+3 t_{m}+3 t_{a}+10 t_{s} ; \\
w_{\text {nea }}=t_{d}+2 t_{m}+2 t_{a}+8 t_{s}+2 t_{s t} ; \\
a_{\text {nеa }}=\left(t_{d}+2 t_{m}+2 t_{a}+8 t_{s}+2 t_{s t}\right) \times 6 / \pi^{2} \\
+\left(t_{d}+t_{m}+t_{a}+5 t_{s}+t_{s t}\right) \times\left(1-6 / \pi^{2}\right)
\end{gathered}
$$

Notice that $a_{\text {xea }}=w_{\text {xea }}$; and

$$
t_{d} \approx t_{m} \gg t_{a} \approx t_{s} \approx t_{s t}
$$

Then (27)-(29) implies that

$$
R:=a_{\text {xea }} / a_{\text {nea }}=2 \pi^{2} /\left(3+\pi^{2}\right)=1.538 \cdots
$$

\section{Conclusions}

Analysis of the proposed algorithm (NEA) for modular multiplicative inverse demonstrates that its execution requires on average $53.8 \%$ less time, than the execution of the Extended Euclid algorithm.

Theoretical analysis of space complexity of the Enhanced-Euclid algorithm shows that it requires relatively small bit-storage for its execution. This storage does not exceed a $2 K$-bit level for a public-key encryption algorithm, where the inputs $p_{0}$ and $p_{1}$ are integers on the interval $\left(10^{100}, 10^{400}\right)$.

On the other hand, computer simulations demonstrate that the average bit-storage is actually $40 \%$ smaller than $2 K$. Hence NEA can be executed if necessary by a custom-built chip with relatively modest memory, [7]. This property of the Enhanced-Euclid algorithm is especially useful for implementation of encryption in low memory environments such as smart or PC cards, cell phones, wearable computers and other integrated devices.

\section{Acknowledgements}

I express my appreciation to R. Rubino, J. Runnells, M. Sikorski, C. Washington and to anonymous reviewer for comments and suggestions that improved the style of this paper; and to A. Koripella for her assistance in running computer experiments.

\section{References}

[1] R. Crandall and C. Pomerance, "Prime Numbers: A Computational Perspective,” Springer, Berlin, 2001.

[2] A. J. Menezes, P. C. van Oorschot and S. A. Vanstone, "Handbook of Applied Cryptography," CRC Press, Boca Raton, 1997.

[3] R. L. Rivest, A. Shamir and L. Adleman, "A Method for Obtaining Digital Signature and Public-Key Cryptosystems," Communications of the ACM, Vol. 21, No. 2, 1978, pp. 120-126.

[4] B. Verkhovsky, "Overpass-Crossing Scheme for Digital Signature,” Keynote Address, Proceedings of International Conference on System Research, Informatics and Cybernetics, Baden-Baden, 30 July-4 August 2001.

[5] T. ElGamal, "A Public-Key Cryptosystem and a Signature Scheme Based on Discrete Logarithms,” IEEE Transactions on Information Theory, Vol. 31, No. 4, 1985, pp. 469-472.

[6] M. Rabin, "Digitized Signatures and Public Key Functions as Intractable as Factorization,” Technical Report: MIT/LCS/TR-212, MIT Laboratory for Computer Science, Cambridge, 1979.

[7] J. Hoffstein, J. Pipher and J. H. Silverman, “An Introduction to Mathematical Cryptography," Springer, Berlin, 2008.

[8] B. Verkhovsky, "Multiplicative Inverse Algorithm and Its Complexity," Proceedings of International ConferenceInterSYMP-99, Baden-Baden, July 1999, pp. 62-67.

[9] B. Verkhovsky, “Accelerated Cyber-Secure Communication Based on Reduced Encryption/Decryption and Information Assurance Protocols," Journal of Telecommunications Management, Vol. 2, No. 3, 2009, pp. 284-293. 
[10] B. Verkhovsky, "Hybrid Authentication Cybersystem Based on Discrete Logarithm, Entanglements and Factorization," International Journal of Communications, Network and System Sciences, Vol. 3, No. 7, 2010, 579-584.

[11] B. Verkhovsky, "Generalized Baby-Step Giant-Step Algorithm for Discrete Logarithm Problem," Advances in Decision Technology and Intelligent Information Systems, International Institute for Advanced Studies in Systems Research and Cybernetics, Baden-Baden, 2008, pp. 8889.

[12] B. Verkhovsky, "Integer Factorization: Solution via Algorithm for Constrained Discrete Logarithm Problem," Journal of Computer Science, 2009, Vol. 5, No. 9, 674-
679.

[13] B. Verkhovsky, "Potential Vulnerability of Encrypted Messages: Decomposability of Discrete Logarithm Problems," International Journal of Communications, Network and System Sciences, Vol. 3, No. 8, 2010, pp. 639-644.

[14] R. B. McClenon, "Leonardo of Pisa and His Liber Quadratorum,” American Mathematical Monthly, Vol. 26, No. 1, 1919, pp. 1-8.

[15] D. Harkin, "On the Mathematical Works of FrancoisEdouard-Anatole Lucas,” Enseignement Mathematique, Vol. 3, No. 2, 1957, pp. 276-288.

\section{Appendix}

\section{Computer experiments}

1) Pairs of $N$ decimal-digit long integers $A$ and $B$ were generated randomly, where $A>B$;

2) Then MMI $C$ of $B$ modulo $A$ was computed; in other words we found an integer $C$, for which holds $B C$ $\bmod A=1$;

3) 125 experiments have been carried out for each value of $N=\{6,8,10, \cdots, 18,20\}$;

4) The values of $S$ (size of stack-storage) for each $N$ were tabulated; (not shown in the Table A1).

5) The values of average $S$ for every $N$ and the range of $S$ for each $N$ are presented in the Table A1.
Table A1. Results of computer experiments.

\begin{tabular}{cccc}
\hline $\boldsymbol{N}$ & Average $\boldsymbol{S}$ & \multicolumn{2}{c}{ Range of $\boldsymbol{S}$} \\
& & {$[\mathbf{m i n}$,} & $\mathbf{m a x}]$ \\
\hline 6 & 12.65 & {$[7$,} & $17]$ \\
8 & 16.20 & {$[9$,} & $21]$ \\
10 & 19.96 & {$[14$,} & $29]$ \\
12 & 24.91 & {$[19$,} & $33]$ \\
14 & 28.53 & {$[18$,} & $36]$ \\
16 & 32.30 & {$[20$,} & $41]$ \\
18 & 36.70 & {$[23$,} & $50]$ \\
20 & 40.29 & {$[26$,} & $54]$ \\
\hline
\end{tabular}

